\title{
Primitive chain network simulations for elongational viscosity of bidisperse polystyrene melts
}

\author{
Keiko Takeda', Sathish K Sukumaran ${ }^{1}$, Masataka Sugimoto ${ }^{1}$, Kiyohito Koyama ${ }^{1}$ and Yuichi Masubuchi ${ }^{2,3^{*}}$
}

${ }^{*}$ Correspondence:

mas@nuap.nagoya-u.ac.jp

${ }^{3}$ Present Address: National Composite Center, Nagoya University, Furocho, Chikusa, Nagoya 464-8603, Japan

Full list of author information is available at the end of the article

\begin{abstract}
In spite of the industrial significance, molecular mechanism of the strain hardening saliently observed in bidisperse polymeric liquids has not been elucidated yet. In this study, the multi-chain slip-link simulation (called primitive chain network simulation) was performed for the bidisperse polystyrene blends for which experimental data for elongational viscosity have been reported earlier. The simulation reasonably reproduced linear viscoelasticity and transient and steady uniaxial elongational viscosities. It has been confirmed that the long chain stretch dominates the stress at the strain hardening as already demonstrated earlier via the tube model. The molecular analysis employing the decoupling approximation revealed for the first time that there exist two molecular mechanisms to induce strain hardening in bidisperse blends. The mechanism switches depending on the Weissenberg number with respect to the Rouse relaxation time of the long chain, $W i_{\mathrm{RL}}$. At $W i_{\mathrm{RL}}<1$, the simultaneous increase of the long chain orientation and stretch with increasing $W_{i_{R L}}$ lifts the viscosity beyond the Trouton's viscosity. At $W i_{R L} \geq 1$, the isotropic short chain suppresses the stretch/ orientation-induced reduction of friction to enhance the stretch of long chain.
\end{abstract}

Keywords: Elongational viscosity, Entangled polymers, Simulation, Strain hardening, Stretch/orientation-induced reduction of friction, Bidisperse system

\section{Background}

Due to the industrial significance, elongational viscosity of polymeric liquids has been widely investigated. In particular, strain hardening, which is the deviation of the elongational viscosity from the linear viscoelastic envelope, has been of industrial and scientific interest because polymeric liquids exhibiting strain hardening are suitable for processing technologies with elongational flow such as fiber spinning, film blowing, blow molding, foaming, etc. [1, 2]. Münstedt [3] measured the uniaxial viscosity for polystyrene melts to report that the strain hardening is intensified when the molecular weight distribution is broadened. Minegishi et al. [4] demonstrated that the small amount of long chain component added to the short chain matrix effectively enhances the strain hardening. Further, Nielsen et al. [5] performed the experiments for the bidispersed polystyrene melts that are mixture of long and short molecules with narrow molecular weight distribution for each. They showed that the steady state viscosity $\eta_{\mathrm{E}}(\dot{\varepsilon})$ is significantly larger than the Trouton's viscosity $3 \eta_{0}$ for the bidispersed samples.

(c) 2015 Takeda et al. This article is distributed under the terms of the Creative Commons Attribution 4.0 International License (http://creativecommons.org/licenses/by/4.0/), which permits unrestricted use, distribution, and reproduction in any medium, provided you give appropriate credit to the original author(s) and the source, provide a link to the Creative Commons license, and indicate if changes were made. 
The molecular mechanism of the strain hardening induced by the molecular weight distribution has not been elucidated yet although in the common consent the stretching of long molecule is suggested as the origin of the viscosity increase [6,7]. Minegishi et al. [4] speculated that the entanglement between long chains induces the long chain stretch. This idea was partly supported by Wagner et al. [8] who analyzed the data to show that the slow relaxation modes dominate the strain hardening. Such slow modes are certainly due to the long chain component. However, the slow modes do not necessarily mean entanglements between long chains. Indeed, the experiments by Nielsen et al. [5] clearly demonstrated that the bidisperse blend exhibits the remarkable strain hardening even if the long chain concentration is lower than the overlapping concentration. Nielsen et al. [5] analyzed their own data by the Wiest model [9] to show that the long chain component dominates the viscosity, as shown by Wagner et al. [8]. However, it is difficult to discuss the molecular origin of the enhanced strain hardening from the anisotropic mobility [10] implemented in the Wiest model. van Ruymbeke et al. [11] utilized the tube model modified with the inter-chain tube pressure effect [12] to analyze the dataset by Nielsen et al. [5]. Their analysis showed that the stretch of the long chain is the origin of the stress at the strain hardening. Although this result was suggested even earlier (as mentioned above), to the author's knowledge their study provided the molecular picture for the first time in the quantitative manner. However, the empirically determined parameter, called the tube diameter relaxation time, complicates the discussion. Namely, they reported that the tube relaxation time for the long chain dissolved in the short chain matrix is larger than that for the monodisperse melt. They stated that the mechanism of this counterintuitive result is an open question. Wagner [13] modified the molecular stress function theory for bidisperse systems with the improved inter-chain tube pressure effect by the dynamic tube dilation. In his theory, the strain hardening for bidisperse system is caused by the enhanced long chain stretch induced by the enlarged tube diameter and the elongated tube relaxation time owing to the tube dilation. However, the coupling between long and short chain components is smeared in the multimode memory function.

In this study, we applied the multi-chain slip-link simulation (called primitive chain network simulation [14]) to the dataset by Nielsen et al. [5] to reveal the molecular mechanism of the strain hardening. We found that there are two mechanisms that enlarge $\eta_{\mathrm{E}}(\dot{\varepsilon})$, and the mechanism switches depending on the Weissenberg number $W_{\mathrm{iRL}}$ with respect to the Rouse relaxation time of the long chain, $\tau_{\mathrm{RL}}$. At $W i_{\mathrm{RL}}<1$, the simultaneous increase of the long chain orientation $S$ and stretch $\lambda$ with increasing $W i_{\mathrm{RL}}$ lifts $\eta_{\mathrm{E}}(\dot{\varepsilon})$ beyond the Trouton's viscosity $3 \eta_{0}$. At $W i_{\mathrm{RL}} \geq 1$, the isotropic short chain suppresses the stretch/orientation-induced reduction of friction (SORF) [15] to enhance the $\lambda$ of the long chain. Details are explained below.

\section{Model and simulation}

Since the model and the code used in this study are common to the previous studies [1517], just a brief description is given below. In the model, a network that consists of network nodes, strands and dangling ends represents the entangled polymer liquid. Each polymer chain is a path between two dangling ends through the consecutive strands (subchains). At each network node, two subchains are bundled by a slip-link so that four subchains diverge 
from each node. The position of the node obeys the Langevin-type equation of motion, which fulfills the force balance among the drag force, subchain tension, osmotic force and Gaussian random force. The sliding motion of the polymer chain through the slip-link is represented by the time development of the number of Kuhn segments on each subchain. In the kinetic equation for the sliding, the force balance is considered between two adjacent subchains for the subchain tension, osmotic force and random force. The network topology is periodically rearranged owing to the hooking/unhooking of the surrounding subchains at the chain ends. Through this topological change, thermal and convective constraint release is naturally considered. The finite chain extensibility was considered with FENE-P approximation where the spring constant was determined by the averaged stretch of the subchains [18]. To reflect the difference of average stretch between short and long components, the FENE constant was set for each component separately. The FENE factor for each component (long and short chains), $f_{\mathrm{FENE}}^{\mathrm{C}}$, is then given as

$$
f_{\mathrm{FENE}}^{\mathrm{C}}=\frac{1}{1-\left\langle\tilde{\lambda}_{\mathrm{C}}^{2}\right\rangle}
$$

Here, the brackets $\langle\cdots\rangle_{\mathrm{C}}$ mean the ensemble average for the segments of each component, and $\tilde{\lambda}$ is the normalized subchain stretch with respect to the full stretch. SORF was considered (unless stated) via the relation between the magnitude of reduction for the Kuhn segment friction (with respect to its equilibrium value) and the stretch/orientation order parameter. We used the empirical equation and its parameters that were determined for the stress relaxation data of monodisperse polystyrene melt [15]. We assume that the Kuhn segment friction is common for the short and long components, and the stretch/orientation order parameter was obtained from the orientation and stretch averaged over the components. The detail of SORF implementation has been published earlier [15-17] and a brief explanation can be found in "Appendix" as well.

Simulations were made for the set of bidisperse polystyrene melts examined by Nielsen et al. [5] and the samples are summarized in Table 1. The unit of molecular weight $M_{0}$ corresponds to the average molecular weight of subchain under equilibrium, and the value for polystyrene melt was set to $11 \mathrm{k}$ as determined in the previous study [19]. Owing to the fluctuation around network nodes in our model, the subchain molecular weight $M_{0}$ is similar to, but different from the entanglement molecular weight $M_{\mathrm{e}}$ that is determined from the plateau modulus via the rubber theory. The empirical relationship we have obtained is $M_{0} \sim 0.6 M_{e}[19,20]$. Nevertheless, once we fix the value of $M_{0}$, the number of subchains for each chain, $Z_{0}=M / M_{0}$, can be determined from the sample molecular weight $M$ accordingly. Here we denote the molecular weight and

Table 1 Examined samples

\begin{tabular}{llll}
\hline & Blend1 & Blend2 & Blend3 \\
\hline$M_{\mathrm{L}}$ & 390,000 & 390,000 & 390,000 \\
$M_{\mathrm{S}}$ & 51,700 & 51,700 & 102,800 \\
$Z_{\mathrm{L}}$ & 35 & 35 & 35 \\
$Z_{\mathrm{S}}$ & 5 & 5 & 9 \\
$\phi_{L}$ & 0.04 & 0.14 & 0.14 \\
\hline
\end{tabular}


the subchain number for long and short chains as $M_{\mathrm{L}}, M_{\mathrm{S}}, Z_{\mathrm{L}}$ and $Z_{\mathrm{S}}$, respectively. The volume fraction of the long chain component is denoted by $\phi_{L}$. The number of Kuhn segments on each subchain (that determines the full stretch for the FENE effect), was determined as $n_{0}=15$ [15-17]. The unit of modulus $G_{0}$ was determined from the relation $G_{0}=\rho R T / M_{0}[19,20]$ as $0.29 \mathrm{MPa}$ at $T=130^{\circ} \mathrm{C}$. The unit time $\tau_{0}$ was determined via fitting for the linear viscoelasticity as $1.0 \mathrm{~s}$. The linear viscoelasticity was calculated from the stress autocorrelation function obtained in quiescent state.

The simulations were made in periodic boundary conditions and the box size for linear viscoelasticity was $16^{3}$ (where the unit length is the average subchain length under equilibrium). The box size for uniaxial elongation was $4 \times 44 \times 44$ and it was stretched to $484 \times 4 \times 4$. This stretch attains the strain of 4.8 and it seems sufficient in comparison to the experimentally accessible value. However, to observe the steady state at $W i_{\mathrm{R}}$ close to unity, we necessarily used the larger box with the dimension of $4 \times 82 \times 82$ and stretched to $1,681 \times 4 \times 4$ for Blend1 and Blend2. This stretch corresponds to the strain of 6.0 .

\section{Results}

\section{Linear viscoelasticity}

Figure 1 shows the linear viscoelasticity of the examined bidisperse blends. With the parameters shown in Table 1 the linear viscoelasticity is reasonably reproduced as earlier reported for the other bidisperse polystyrene melts [21]. In particular, for Blend1 the second plateau (at $\omega \sim 2 \times 10^{-3} \mathrm{rad} / \mathrm{s}$ ) is correctly captured. On the other hand, for the other two samples the simulation shows the faster relaxation than experiment so that the longest relaxation time is somewhat underestimated.

\section{Transient elongational viscosity}

Figure 2 shows the transient viscosity $\eta_{\mathrm{E}}^{+}(\dot{\varepsilon}, t)$. The simulation nicely captures $\eta_{\mathrm{E}}^{+}(\dot{\varepsilon}, t)$ at $\dot{\varepsilon}>1 / \tau_{\mathrm{RL}}=0.016 \mathrm{~s}^{-1}$ (where $\tau_{\mathrm{RL}}$ is the viscoelastic Rouse relaxation time of the long chain calculated from the relation $\tau_{\mathrm{R}}=Z_{L}^{2} \tau_{0} / 2 \pi^{2}$ as derived earlier [22]). For example, for $\eta_{\mathrm{E}}^{+}(\dot{\varepsilon}, t)$ of Blend1 the simulation result (red curve) at $\dot{\varepsilon}=0.3 \mathrm{~s}^{-1}$ (the leftmost curve) almost coincides with the data (symbol). Fair coincidence can be seen for all the blends at $\dot{\varepsilon}=0.3,0.1$ and $0.03 \mathrm{~s}^{-1}$. (Note that for Blend2 $\eta_{\mathrm{E}}^{+}(\dot{\varepsilon}, t)$ at $\dot{\varepsilon}=0.3 \mathrm{~s}^{-1}$ is not available in the literature.) On the other hand, the simulation underestimates the data at $\dot{\varepsilon} \leq 1 / \tau_{\mathrm{RL}}$. In particular, at $\dot{\varepsilon}=0.003$ and $0.001 \mathrm{~s}^{-1}, \eta_{\mathrm{E}}^{+}(\dot{\varepsilon}, t)$ from the simulation is close to the linear viscoelastic envelope (black dotted curve, obtained from the simulated $G^{\prime}$ and $G^{\prime \prime}$ ) but the data show clear deviations from the linear viscoelastic response. This discrepancy in $\eta_{\mathrm{E}}^{+}(\dot{\varepsilon}, t)$ between the data and simulation results is partly attributable to the discrepancy in $G^{\prime}$ and $G^{\prime \prime}$, in which the simulation predicts faster relaxation than experiment for Blend2 and Blend3 as mentioned for Figure 1. However, the discrepancy in $\eta_{\mathrm{E}}^{+}(\dot{\varepsilon}, t)$ is also seen for Blend1, for which $G^{\prime}$ and $G^{\prime \prime}$ are quantitatively reproduced. The mechanism of this discrepancy is unknown.

Concerning the effect of SORF, the simulations with and without SORF exhibited no difference in $\eta_{\mathrm{E}}^{+}(\dot{\varepsilon}, t)$, except for Blend3 at $\dot{\varepsilon}=0.3 \mathrm{~s}^{-1}$ (the leftmost curve). At this 


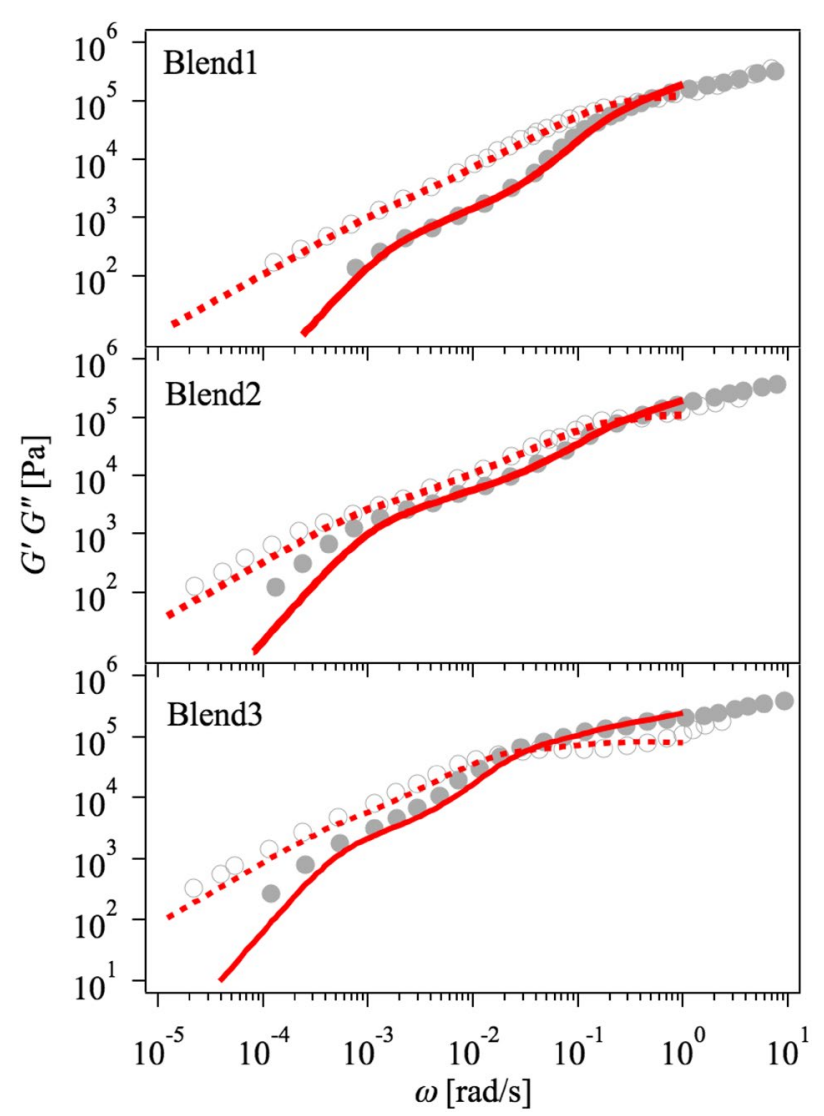

Figure 1 Linear viscoelasticity for Blend1, Blend2 and Blend3 from top to bottom. Filled and unfilled circles are $G^{\prime}$ and $G^{\prime \prime}$ obtained by Nielsen et al. [5]. Red solid and dotted curves are simulation results.

condition, the simulated $\eta_{\mathrm{E}}^{+}(\dot{\varepsilon}, t)$ without SORF (blue curve) is significantly higher than the result with SORF (red curve). In comparison to the monodisperse melts, in which SORF appears to reduce the viscosity at $\dot{\varepsilon}>1 / \tau_{\mathrm{R}}$, in the bidisperse blends the activation of SORF is suppressed by the short chains, as discussed later. Nevertheless, the simulation with SORF reproduces the data better than that without SORF.

\section{Steady state elongational viscosity}

Figure 3 shows the steady state viscosity $\eta_{\mathrm{E}}(\dot{\varepsilon})$. As mentioned for $\eta_{\mathrm{E}}^{+}(\dot{\varepsilon}, t)$, the simulation reasonably captures the data at $\dot{\varepsilon}>1 / \tau_{\mathrm{RL}}$ whereas it underestimates the data at $\dot{\varepsilon} \leq 1 / \tau_{\mathrm{RL}}$. The simulated $\eta_{\mathrm{E}}(\dot{\varepsilon})$ (red and blue curves) is consistent with the linear viscoelasticity at low $\dot{\varepsilon}$; the simulated $\eta_{\mathrm{E}}(\dot{\varepsilon})$ (blue and red curves) converges to $3 \eta_{0}$ (dotted line) around $\dot{\varepsilon}=1 / \tau_{1}$. On the other hand, the experimental $\eta_{\mathrm{E}}(\dot{\varepsilon})$ is higher than $3 \eta_{0}$ suggesting some slow relaxation modes for which the relaxation time is longer than the simulated $\tau_{1}$. The simulation failed to predict these behaviors by unknown reasons. The effect of SORF (difference between red and blue curves) appears only at $\dot{\varepsilon}$ which is well-beyond $1 / \tau_{\mathrm{RL}}$, as mentioned for $\eta_{\mathrm{E}}^{+}(\dot{\varepsilon}, t)$. 


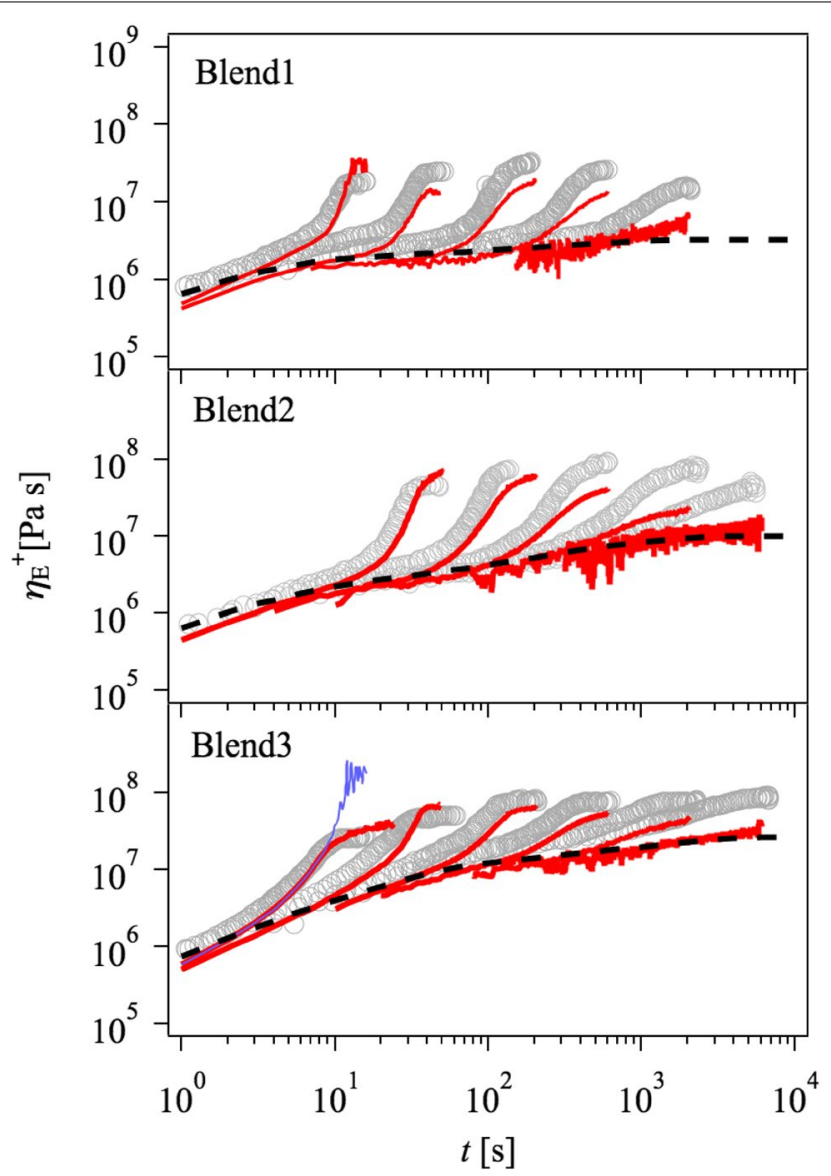

Figure 2 Transient uniaxial elongational viscosities for Blend1, Blend 2 and Blend 3 from top to bottom. The strain rates (from left to right) are $0.3,0.1,0.03,0.01$ and $0.003 \mathrm{~s}^{-1}$ for Blend1, 0.1, 0.03, 0.01, 0.003 and $0.001 \mathrm{~s}^{-1}$ for Blend2, and $0.3,0.1,0.03,0.01,0.003$ and $0.001 \mathrm{~s}^{-1}$ for Blend3, respectively. Unfilled circle is the data measured by Nielsen et al. [5] (the data for Blend2 were taken from van Ruymbeke et al. [11]). Red and blue solid curves are simulation results with and without SORF. Black dotted curve is the linear viscoelastic envelope for the simulation.

\section{Decoupling analysis on the role of the long chain}

In this section, we attempt molecular level analysis on the steady state viscosity for Blend1 to reveal the significance of the long chain contribution on the basis of the decoupling approximation [18] in which the stress is decomposed as

$$
\sigma \approx\left(\frac{Z}{Z_{0}}\right) f_{\mathrm{FENE}} \lambda^{2} S
$$

Here, $Z$ is the average number of subchains and $Z_{0}$ is its equilibrium value, $f_{\mathrm{FENE}}$ is the FENE spring constant, $\lambda$ is the stretch normalized with respect to the maximum stretch, and $S$ is the subchain orientation defined as $S=u_{x}^{2}-u_{y}^{2}$ (where $\mathbf{u}$ is the subchain orientation vector and $x$ and $y$ are the stretch and normal directions, respectively). The numerical prefactor is neglected here. Figure 4 shows the decoupled measures $\left(Z / Z_{0}\right.$, $S, \lambda^{2}$ and $f_{\mathrm{FENE}}$ ) plotted against $\dot{\varepsilon}$. In the top panel, as shown in the earlier studies, the total viscosity (black curve) is dominated by the minor long chain component (red solid curve), and the short chain contribution (red dotted curve) is relatively small. In 


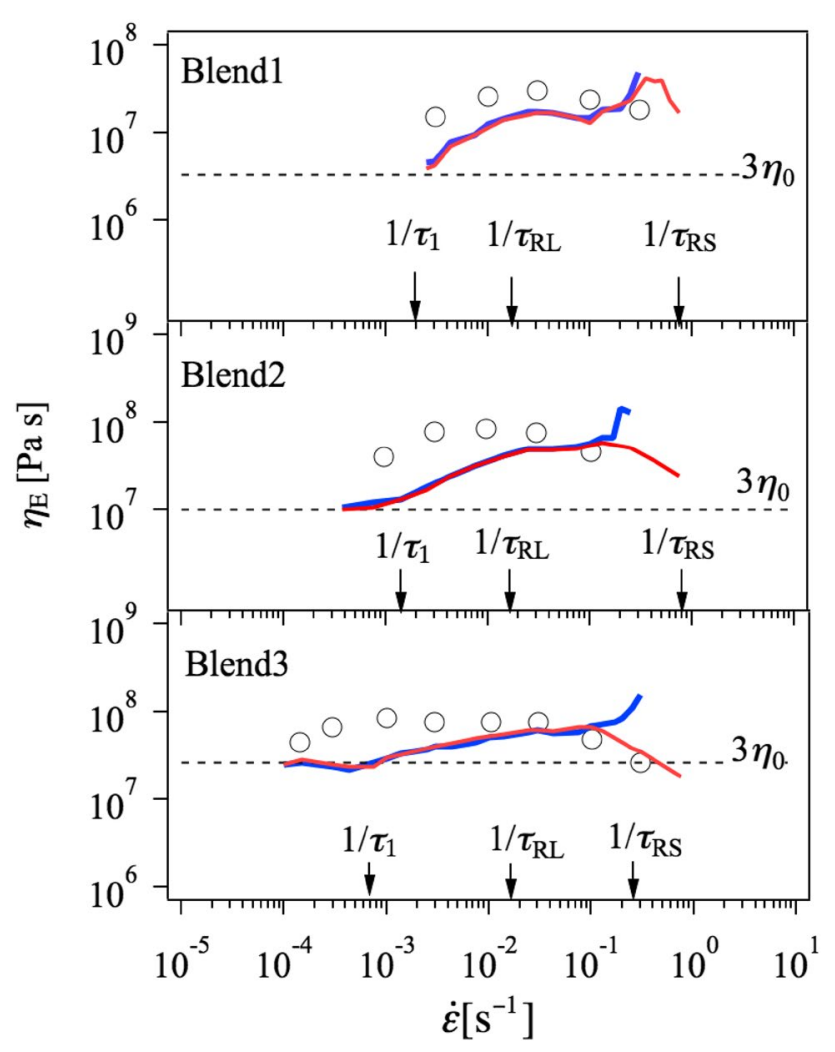

Figure 3 Steady state viscosity as a function of strain rate for Blend1, Blend2 and Blend3 from top to bottom. Red and blue curves are the simulation results with and without SORF. Unfilled circle is the data extracted from Nielsen et al. [5]. Horizontal dotted line is the Trouton's viscosity. The reciprocal of the longest relaxation time $\tau_{1}$, the (viscoelastic) Rouse relaxation times of the long and short chains, $\tau_{\mathrm{RL}}$ and $\tau_{\mathrm{RS}}$, are also indicated at the horizontal axis.

the bottom panel, the reduced friction with respect to the equilibrium value, $\zeta / \zeta(0)$, is shown for comparison.

The behavior of molecular measures shown in Figure 4 can be divided into five regimes with respect to $\dot{\varepsilon}$. In the low strain rate limit ( $\dot{\varepsilon}<1 / \tau_{1}$ : Region I) $S$ linearly increases with increasing $\dot{\varepsilon}$ with no stretch both for long and short chains. This linearity of $S$ appears in the viscosity that maintains the Trouton's viscosity. At the strain rates located in the range $1 / \tau_{1}<\dot{\varepsilon}<1 / \tau_{\mathrm{RL}}$ (Region II), the stretch of the long chain occurs in addition to the linear growth of $S$. This simultaneous growths of $S$ and $\lambda$ induce the increase of $\eta_{\mathrm{E}}(\dot{\varepsilon})$, and consequently $\eta_{\mathrm{E}}(\dot{\varepsilon})$ is lifted up beyond $3 \eta_{0}$ to show the strain hardening. (One may argue that the long chain stretch should be observed at $\dot{\varepsilon}>1 / \tau_{\mathrm{RL}}$, and not at the lower strain rates. However, the stretch transition is not sharp and the stretch gradually starts from lower $\dot{\varepsilon}$, as discussed for elastic dumbbells [6]. It is also noted that the $\tau_{\mathrm{RL}}$ value discussed here is the viscoelastic Rouse relaxation time that is $1 / 2$ of the end-to-end relaxation time. For the monodisperse melt of long chain exhibits similar stretching behavior as shown later.) Interestingly, the short chain viscosity also increases with increasing $\dot{\varepsilon}$ even without stretch. This increase of short chain viscosity is due to the coupling between long and short chains through entanglement. Nevertheless, the short chain contributes much less than the long chain. 


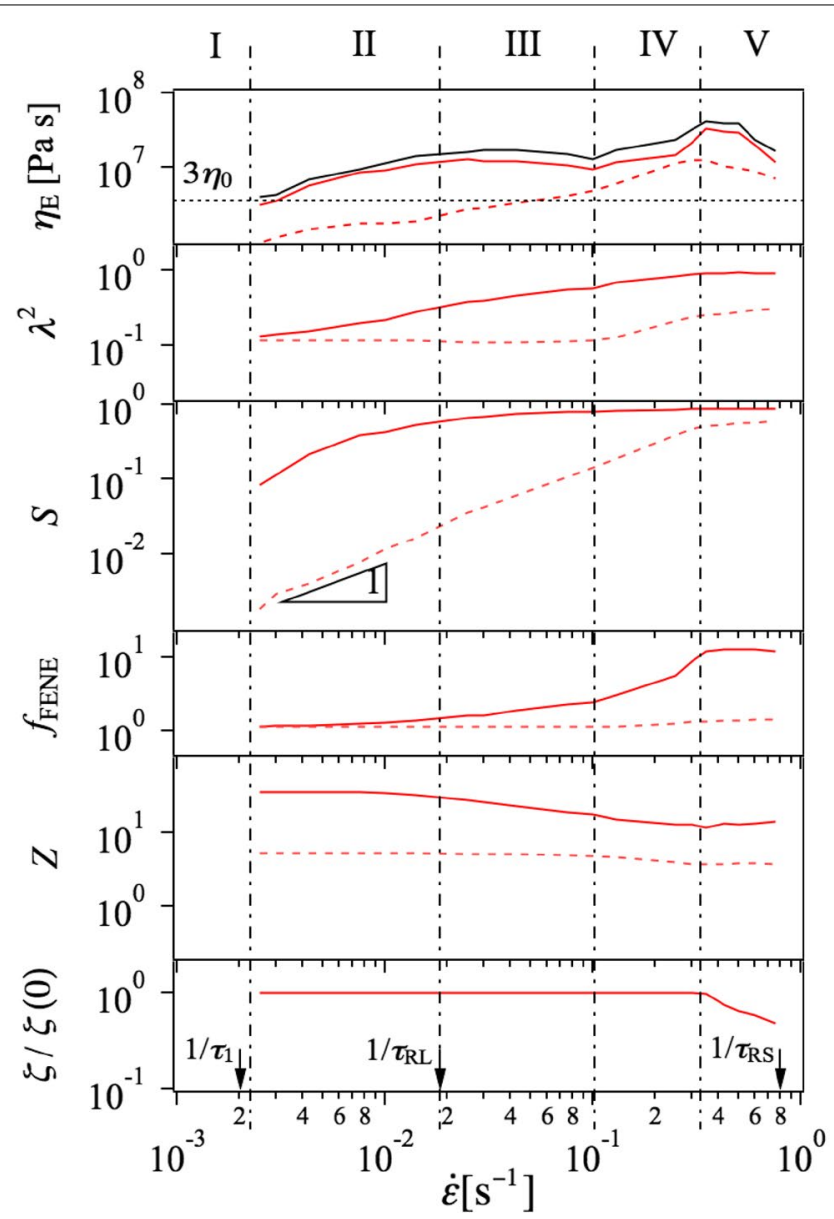

Figure 4 Steady state viscosity $\eta_{E^{\prime}}$ squared reduced stretch $\lambda^{2}$, polymer anisotropy $S$, FENE factor $f_{\text {FENE, }}$, entanglement segment number per chain $Z$, and reduced friction $\zeta / \zeta(0)$ for Blend1 (from top to bottom) plotted against strain rate $\dot{\varepsilon}$. Solid and dotted red curves are for long and short chains. Black curve and horizontal dotted line in the top panel are the total viscosity and the Trouton's viscosity, respectively. Dot dashed vertical lines indicate the borders between different regions (see text for detail). The reciprocal of the longest relaxation time $\tau_{1}$, the (viscoelastic) Rouse relaxation times of the long and short chains, $\tau_{\mathrm{RL}}$ and $\tau_{\mathrm{RS}}$, are also indicated at the horizontal axis.

In the higher strain rate range $\left(1 / \tau_{\mathrm{RL}}<\dot{\varepsilon}\right)$, there exist two critical strain rates. One of the critical rates is located around $10^{-1} \mathrm{~s}^{-1}$ beyond that the short chain stretches (see dotted line in the 2nd panel). The other one is around $3 \times 10^{-1} \mathrm{~s}^{-1}$ beyond that the friction decreases due to SORF (see bottom panel). These critical strain rates are indicated as dash-dotted vertical lines in Figure 4 to classify the regions III-V. At the strain rates in the range $1 / \tau_{\mathrm{RL}}<\dot{\varepsilon}<10^{-1} \mathrm{~s}^{-1}$ (Region III), the growth rate of $S$ for the long chain is declined as $S$ approaches to the maximum value ( $S \leq 1$ by definition). The growths of $\lambda$ and $f_{\mathrm{FENE}}$ for the long chain retain the viscosity constant. The linear growth of the short chain viscosity is maintained, even though the short chain stretch does not occur in this region, but the contribution is still small. On the other hand, in the Region IV $\left(10^{-1} \mathrm{~s}^{-1}<\dot{\varepsilon}<3 \times 10^{-1} \mathrm{~s}^{-1}\right)$, as the short chain stretch grows, the short chain contribution in the viscosity also grows to be comparable to the long chain contribution. The long chain viscosity increases mainly owing to the increase of $f_{\mathrm{FENE}}$. In the highest strain 
rate region (Region V), SORF occurs due to the stretch/orientation of the short chains that dominates the stretch/orientation order parameter of the system as a whole.

\section{Comparison of the long chain behavior to the monodisperse system}

To illuminate the difference of the long chain behavior between monodisperse and bidisperse systems, the decoupled measures for both cases are shown in Figure 5 as functions of the Weissenberg number $W i_{\mathrm{RL}}$ defined with respect to $\tau_{\mathrm{RL}}$. The subchain number is omitted for simplicity.

In the slow flow regime $\left(W i_{\mathrm{RL}}<1\right)$, the remarkable difference is observed in the growth of $S$ (3rd panel). Although it is common for both cases that the $S$ growth starts at $\dot{\varepsilon} \approx 1 / \tau_{1}$, the shortened $\tau_{1}$ of the bidisperse system due to the short chains retards the $S$ growth (red solid curve) in comparison to that of the monodisperse case (black dotted curve). Interestingly, $S$ for the bidisperse system steeply increases to reach a similar value to the monodisperse system at $W i_{\mathrm{RL}}=1$. As a consequence, the bidisperse system exhibits the simultaneous growth of $S$ and $\lambda$, which causes the strain hardening $\left(\eta_{\mathrm{E}}>3 \eta_{0}\right)$. On the other hand, for the monodisperse system the $\lambda$ growth separately occurs after the $S$ growth. Since the growth rates for $\lambda$ and $S$ against $\dot{\varepsilon}$ are less than the linear relationship, strain softening occurs $\left(\eta_{\mathrm{E}}<3 \eta_{0}\right)$. Note that the critical $W i_{\mathrm{RL}}$ value at which $\lambda$ starts growing is around $2 \times 10^{-1}$ and it is common for the monodisperse and bidisperse systems. (As mentioned in the previous section, it is noted that the stretch observed at the strain rates lower than $W i_{\mathrm{RL}}=1$ is just due to the nature of the Rouse chain [6].) The growth rate of $\lambda$ is also similar to each other in $W i_{\mathrm{RL}}<1$.

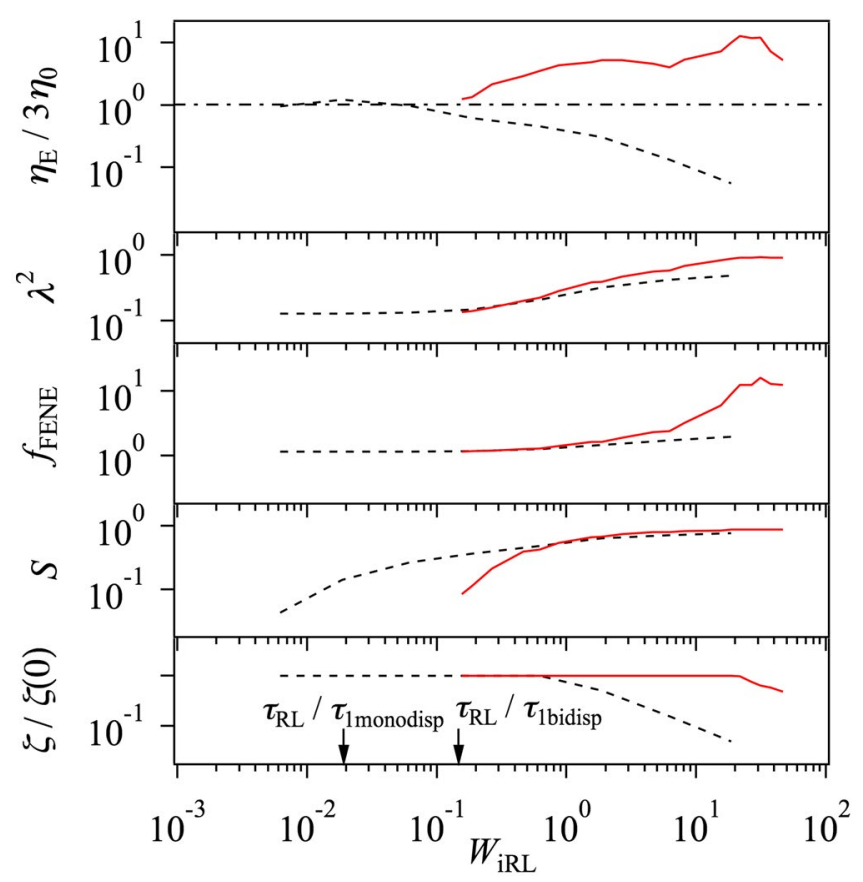

Figure 5 Steady state viscosity, squared reduced stretch, FENE factor, orientation and reduced friction for the long chain as functions of $\tau_{\mathrm{RL}}$ based Weissenberg number $W i_{\mathrm{RL}}$ in Blend1 (red solid curve) and monodisperse system (black broken curve). The longest relaxation times, $\tau_{1 \text { monodisp' }}$ and $\tau_{1 \text { bidisp }}$ are indicated at the horizontal axis. 
It is fair to mention that the simultaneous growth of $S$ and $\lambda$ for bidisperse systems has been suggested earlier (but rather implicitly). Wagner [13] analyzed the dataset of Nielsen et al. [5] by the molecular stress function theory to report that the strain hardening is induced by the dynamic tube dilation through the reduction of the inter-chain tube pressure. Although Wagner did not discuss the effect of long chain orientation, his picture is essentially common with ours in a sense that the reduced motional constraint for the long chain induces the strain hardening.

In the fast flow region ( $\left.W i_{\mathrm{RL}} \geq 1\right)$, the $S$ growth saturates and $\lambda$ growth dominates the $\eta_{\mathrm{E}}$ behavior. In this respect, the remarkable difference between the monodisperse and bidisperse systems is the appearance of SORF. See bottom panel, in which the friction decreases with increasing $\dot{\varepsilon}$ for the monodisperse system (black dotted curve) whereas it retains the equilibrium value for the bidisperse system (red solid curve) up to the much higher value of $W i_{\mathrm{RL}}(\sim 20)$. The difference in friction is induced by the total stretch/ orientation of the system. For the monodisperse system, the high stretch/orientation of the system as a whole induces SORF. This occurrence of SORF suppressed $\lambda$ and $f_{\text {FENE}}$, and then the monotonic decrease of $\eta_{\mathrm{E}}$ is attained. On the contrary, for the bidisperse system, the average stretch/orientation are maintained at low values due to the short chains (that is not stretched/oriented at this flow rate). Then the growth rate of $\lambda$ against $\dot{\varepsilon}$ is close to the linear relationship, which compensates the declined growth rate of $S$ and maintains $\eta_{\mathrm{E}}>3 \eta_{0}$.

\section{Discussion}

The decoupling analysis (shown in Figures 4,5) has revealed that the strain hardening in bidisperse blends is induced by the simultaneous growth of orientation and stretch of the long chain (at $W i_{\mathrm{RL}}<1$ ) and the suppressed SORF due to the short chain (at $W i_{\mathrm{RL}} \geq 1$ ). In this section, this explanation shall be evaluated for the other systems.

Let us consider the effect of molecular weight on the strain hardening for monodisperse linear polymers. The simultaneous growth of orientation and stretch is induced when the longest (orientational) relaxation time and the Rouse (stretch) relaxation time are not separated from each other. The ratio of two relaxation times is reduced if the number of entanglement per molecule is small. Indeed, Nielsen et al. [5] reported that for the polystyrene melt with relatively low molecular weight $(M=51.7 \mathrm{k})$ shows $\eta_{\mathrm{E}}>3 \eta_{0}$ whereas the higher molecular weight samples $(M=102.8 \mathrm{k}, 200 \mathrm{k}$ and $390 \mathrm{k})$ exhibit $\eta_{\mathrm{E}}<3 \eta_{0}$. Concerning the effect of SORF at $W i_{\mathrm{RL}} \geq 1$, it has been demonstrated that SORF is suppressed and intensive strain hardening occurs if polymer concentration is low (regardless of the amount of entanglement in the system) [15, 17]. These results suggest that the strain hardening is intensified for less entangled system. This view is consistent with the fact that the (unentangled) dumbbell models exhibit clear strain hardening [6].

Let us move to multi-branched polymers for which intensive strain hardening has been reported [23]. For such polymers, because the diffusion of the branch point dominates both of orientation and contraction relaxation times of the backbone [24], these two characteristic times are not well-separated. Consequently, the simultaneous growth of orientation and stretch occurs, and $\eta_{\mathrm{E}}$ becomes larger than $3 \eta_{0}$ at relatively low $\dot{\varepsilon}$, as shown earlier [16]. On the other hand, the backbone stretch (and the corresponding 
strain hardening) at high $\dot{\varepsilon}$ is dominated by the occurrence of the branch point withdrawal [25], which is the other important relaxation mechanism.

\section{Conclusion}

The PCN simulations have been performed for bidisperse polystyrene melts for which Nielsen et al. [5] have reported the uniaxial elongational viscosity experimentally. The simulation reasonably reproduced linear viscoelasticity and transient and steady uniaxial elongational viscosities, though some inconsistencies between the data and simulations were found due to unknown reasons. The molecular analysis based on the decoupling approximation was performed to reveal the mechanism of strain hardening in the bidisperse systems. The long chain stretch dominates the stress at the strain hardening, as already demonstrated earlier via the tube models. On the other hand, the induction mechanism of the strain hardening has been clarified for the first time. At the strain rates of $W i_{\mathrm{RL}}<1$, the simultaneous growth of orientation and stretch of the long chain causes the non-linear growth of stress and the raise of viscosity beyond the linear value. This mechanism is essentially the same for the elastic dumbbell for which stretch and orientational relaxations are not separated. Nevertheless, it is worth noting that the long chain behavior observed in this study is not reproduced unless the orientation coupling between the chains is correctly captured through thermal and convective release. At the strain rates of $W i_{\mathrm{RL}} \geq 1$, the suppression of SORF by the short chain component (that exhibits relatively low stretch and orientation) maintains the stretch of long chain.

\section{Appendix}

Although SORF has been reported earlier [15] and the implementation to the primitive chain network simulation has been described [15-17], we present a brief description in this appendix for readers' convenience. Yaoita et al. [15] have found the acceleration of the stress relaxation after uniaxial stretch. The magnitude of the acceleration depends on the magnitude of polymer stretch and orientation at the initial state of the relaxation. They organized the results into an empirical relationship between the polymer friction and the stretch/orientation order parameter as shown below.

$$
\begin{aligned}
& \frac{\zeta\left(F_{\mathrm{SO}}^{\prime}\right)}{\zeta(0)}=f_{\mathrm{FENE}} \frac{1}{(1+\beta)^{\gamma}}\left[\beta+\frac{1}{2}\left\{1-\tanh \alpha\left(F_{\mathrm{SO}}^{\prime}-F_{\mathrm{SO}}^{\prime *}\right)\right\}\right]^{\gamma} \\
& F_{\mathrm{SO}}^{\prime}=F_{\mathrm{SO} f_{\mathrm{FENE}}} \\
& F_{\mathrm{SO}}=\tilde{\lambda}^{2} S
\end{aligned}
$$

$\zeta$ is the segmental friction and $\zeta(0)$ is the equilibrium value. $F_{\mathrm{SO}}$ is the stretch/orientation order parameter. $f_{\mathrm{FENE}}$ is the FENE factor (Eq. 1) but averaged for the system as a whole, not for each component. $\alpha(=20), \beta\left(=5 \times 10^{-9}\right), \gamma(=0.15)$ and $\mathrm{F}_{\mathrm{SO}}^{\prime *}(=0.14)$ are the parameters that were determined via fitting of the experimental data [15]. In this present study, we implemented the SORF relationships (Eqs. 3-5) into the primitive chain network simulation. We calculate the value of $F_{\mathrm{SO}}$ at each simulation timestep from the subchain vectors to obtain $\zeta / \zeta(0)$. Then, the calculation at $t+\Delta t$ is performed 
with respect to the value of $\zeta / \zeta(0)$ at time $t$. Note that we do not change the friction coefficient in the kinetic equations to avoid violation of the fluctuation-dissipation relationship for each segment. Instead, we modulate the flow rate to keep the Weissenberg number that varies according to the change of $\zeta / \zeta(0)$. The primitive chain network simulations with the SORF implementation mentioned above has attained semi-quantitative agreement with experiments for entangled polymer solutions and melts of linear polymers $[15,17]$ and a pom-pom branch polymer [16]. It is noteworthy that the same SORF relationship has been examined via the tube model as well [26].

\section{Authors' contributions}

YM performed the code development, simulations, data acquisition, analysis, constructing the discussion and paper writing. KT helped the simulations and the paper writing. SKS, MS and KK contributed the discussion. All authors read and approved the final manuscript.

\section{Author details}

${ }^{1}$ Graduate School of Science and Engineering, Yamagata University, Yonezawa 992-8510, Japan. ${ }^{2}$ Institute for Chemical Research, Kyoto University, Kyoto 611-0011, Japan. ${ }^{3}$ National Composite Center, Nagoya University, Furocho, Chikusa, Nagoya 464-8603, Japan.

\section{Acknowledgements}

YM is financially supported by Grant-in-Aid for Scientific Research (B) No. 26288059 from JSPS.

\section{Compliance with ethical guidelines}

Competing interest

The authors declare that they have no competing interests.

Received: 27 February 2015 Accepted: 2 June 2015

Published online: 18 June 2015

\section{References}

1. Cogswell FN (1969) Tensile deformations in molten polymers. Rheol Acta 8:187-194. doi:10.1007/BF01984657

2. Spitael P, Macosko CW (2004) Strain hardening in polypropylenes and its role in extrusion foaming. Polym Eng Sci 44:2090-2100. doi:10.1002/pen.20214

3. Münstedt H (1980) Dependence of the elongational behavior of polystyrene melts on molecular weight and molecular weight distribution. J Rheol 24:847. doi:10.1122/1.549587

4. Minegishi A, Nishioka A, Takahashi T, Masubuchi Y, Takimoto J, Koyama K (2001) Uniaxial elongational viscosity of PS/a small amount of UHMW-PS blends. Rheol Acta 40:329-338. doi:10.1007/s003970100165

5. Nielsen JK, Rasmussen HK, Hassager O, McKinley GH (2006) Elongational viscosity of monodisperse and bidisperse polystyrene melts. J Rheol 50:453-476. doi:10.1122/1.2206711

6. Bird RB, Curtiss CF, Armstrong RC, Hassager O (1987) Dynamics of polymeric liquids. In: Kinetic theory, vol 2. Wiley, New York

7. Marrucci G, Grizzuti N (1988) Fast flows of concentrated polymers: predictions of the tube model on chain stretching. Gazz Chim Ital 118:179-185

8. Wagner MH, Kheirandish S, Koyama K, Nishioka A, Minegishi A, Takahashi T (2004) Modeling strain hardening of polydisperse polystyrene melts by molecular stress function theory. Rheol Acta 44:235-243. doi:10.1007/ s00397-004-0402-7

9. Wiest JM (1989) A differential constitutive equation for polymer melts. Rheol Acta 28:4-12. doi:10.1007/BF01354763

10. Giesekus H (1982) A simple constitutive equation for polymer fluids based on the concept of deformation-dependent tensorial mobility. J Non Newton Fluid Mech 11:69-109. doi:10.1016/0377-0257(82)85016-7

11. van Ruymbeke E, Nielsen J, Hassager O (2010) Linear and nonlinear viscoelastic properties of bidisperse linear polymers: mixing law and tube pressure effect. J Rheol 54:1155. doi:10.1122/1.3478316

12. Marrucci G, lanniruberto $G$ (2004) Interchain pressure effect in extensional flows of entangled polymer melts. Macromolecules 37:3934-3942. doi:10.1021/ma035501u

13. Wagner MH (2011) The effect of dynamic tube dilation on chain stretch in nonlinear polymer melt rheology. J Non Newton Fluid Mech 166:915-924. doi:10.1016/j.jnnfm.2011.04.006

14. Masubuchi Y, Takimoto J, Koyama K, lanniruberto G, Marrucci G, Greco F (2001) Brownian simulations of a network of reptating primitive chains. J Chem Phys 115:4387-4394. doi:10.1063/1.1389858

15. Yaoita T, Isaki T, Masubuchi Y, Watanabe H, lanniruberto G, Marrucci G (2012) Primitive chain network simulation of elongational flows of entangled linear chains: stretch/orientation-induced reduction of monomeric friction. Macromolecules 45:2773-2782. doi:10.1021/ma202525v

16. Masubuchi Y, Matsumiya Y, Watanabe H, Marrucci G, lanniruberto G (2014) Primitive chain network simulations for pom-pom polymers in uniaxial elongational flows. Macromolecules 47:3511-3519. doi:10.1021/ma500357g 
17. Masubuchi Y, Matsumiya Y, Watanabe H (2014) Test of orientation/stretch-induced reduction of friction via primitive chain network simulations for polystyrene, polyisoprene, and poly( $n$-butyl acrylate). Macromolecules 47:140924145911000. doi:10.1021/ma5016165

18. Yaoita T, Isaki T, Masubuchi Y, Watanabe H, lanniruberto G, Marrucci G (2011) Primitive chain network simulation of elongational flows of entangled linear chains: role of finite chain extensibility. Macromolecules 44:9675-9682. doi:10.1021/ma202166y

19. Masubuchi Y, lanniruberto G, Greco F, Marrucci G (2008) Quantitative comparison of primitive chain network simulations with literature data of linear viscoelasticity for polymer melts. J Non Newton Fluid Mech 149:87-92. doi:10.1016/j.jnnfm.2007.02.014

20. Masubuchi Y, lanniruberto G, Greco F, Marrucci G (2003) Entanglement molecular weight and frequency response of sliplink networks. J Chem Phys 119:6925-6930. doi:10.1063/1.1605382

21. Masubuchi Y, Watanabe H, lanniruberto G, Greco F, Marrucci G (2008) Comparison among slip-link simulations of bidisperse linear polymer melts. Macromolecules 41:8275-8280. doi:10.1021/ma800954q

22. Furuichi K, Nonomura C, Masubuchi Y, Watanabe H, lanniruberto G, Greco F et al (2008) Entangled polymer orientation and stretch under large step shear deformations in primitive chain network simulations. Rheol Acta 47:591599. doi:10.1007/s00397-008-0258-3

23. Münstedt H, Laun HM (1981) Elongational properties and molecular structure of polyethylene melts. Rheol Acta 20:211-221. doi:10.1007/BF01678022

24. Masubuchi Y, lanniruberto G, Greco F, Marrucci G (2006) Primitive chain network simulations for branched polymers. Rheol Acta 46:297-303. doi:10.1007/s00397-006-0112-4

25. McLeish T, Larson R (1998) Molecular constitutive equations for a class of branched polymers: the pom-pom polymer. J Rheol 42:81-110. doi:10.1122/1.550933

26. Yaoita T, Masubuchi Y, Watanabe H (2014) Concept of stretch/orientation-induced friction reduction tested with a simple molecular constitutive equation. Nihon Reoroji Gakkaishi 42:207-213. doi:10.1678/rheology.42.207

\section{Submit your manuscript to a SpringerOpen ${ }^{\circ}$ journal and benefit from:}

- Convenient online submission

- Rigorous peer review

- Immediate publication on acceptance

- Open access: articles freely available online

- High visibility within the field

- Retaining the copyright to your article

Submit your next manuscript at $>$ springeropen.com 